\title{
Information Theoretic Criteria for Community Detection
}

\author{
L. Karl Branting \\ The MITRE Corporation, \\ 7525 Colshire Drive \\ McLean, VA 22102, USA \\ lbranting@mitre.org
}

\begin{abstract}
Many algorithms for finding community structure in graphs search for a partition that maximizes modularity. However, recent work has identified two important limitations of modularity as a community quality criterion: a resolution limit; and a bias towards finding equal-sized communities. Information-theoretic approaches that search for partitions that minimize description length are a recent alternative to modularity. This paper shows that two information-theoretic algorithms are themselves subject to a resolution limit, identifies the component of each approach that is responsible for the resolution limit, proposes a variant, SGE (Sparse Graph Encoding), that addresses this limitation, and demonstrates on three artificial data sets that (1) SGE does not exhibit a resolution limit on sparse graphs in which other approaches do, and that (2) modularity and the compression-based algorithms, including SGE, behave similarly on graphs not subject to the resolution limit.
\end{abstract}

\section{Introduction}

Many complex networks, such as the Internet, metabolic pathways, and social networks, are characterized by a community structure that groups related vertices together. Traditional clustering techniques group vertices based on some metric for attribute similarity [2]. More recent research has focused on detection of community structure from graph topology. Under this approach, the input to a community-detection algorithm is a graph in which vertices correspond to individuals (e.g., URLs, molecules, or people) and edges correspond to relationships (e.g., hyperlinks, chemical reactions, or marital and business ties). The output consists of a partition of the graph in which subgraphs correspond to meaningful groupings (e.g., web communities, families of molecules, or social clans). ${ }^{1}$

Community detection algorithms can be viewed as comprising two components: a utility function that expresses the quality of any given partition of a

\footnotetext{
${ }^{1}$ Some communities, such as social clubs and families, can overlap. Membership in such communities is better modeled as attributes of vertices rather than through a partition of the graph [3]. The focus of this paper, however, as in the bulk of community detection research, is on partition-based community structure.
} 


\begin{tabular}{|l|l|l|}
\hline utility function & search strategy & algorithm \\
\hline \hline modularity & DHC/betweenness centrality & Newman \& Girvan (2004) [11] \\
\hline modularity & AHC & Newman (2004) [1] \\
\hline modularity & Genetic Algorithm & Tasgin \& Bingol (2006) [12] \\
\hline modularity & DHC/network structure index & Rattigan et al. (2007) [13] \\
\hline modularity & AHC/spectral division & Donetti \& Munoz (2004) [14] \\
\hline log-likelihood & fixed-point iteration & Zhang et al. (2007) [15] \\
\hline MDL & simulated annealing & Rosvall \& Bergstrom (2007) [16] \\
\hline MDL & iterated hill climbing & Chakrabarti (2004) [8] \\
\hline
\end{tabular}

Table 1. Utility functions and search strategies for various community-detection algorithms. $D H C$ represents divisive hierarchical clustering, $A D H H$ represents agglomerative hierarchical clustering, and $M D L$ represents "Minimum Description length."

graph; and a search strategy that specifies a procedure for finding a partition that optimizes the utility function. Table 1 sets forth utility functions and search strategies of eight recent community-detection algorithms, showing that utility functions have been paired with a variety of different search strategies.

The utility function most prevalent in recent community detection research is the modularity function introduced in [1]:

$$
Q=\sum_{1<i \leq m}\left(w\left(D_{i i}\right) / l-\left(l_{i} / l\right)^{2}\right)
$$

where $i$ is the index of the communities, $w\left(D_{i i}\right)$ is the number of edges in the graph that connect pairs of vertices within community $i, l_{i}=\sum_{j \leq i} w\left(D_{i j}\right)$, i.e., the number of edges in the graph that are incident to at least one vertex in community $i$, and $l$ is the total number of edges in the entire graph. Modularity formalizes the intuition that communities consist of groups of entities having more links with each other than with members of other groups.

Because of the shortage of real-world data sets with known community structure, maximum modularity has sometimes even been equated with correct community structure. However, two important weaknesses have been identified in modularity as a community-structure criterion.

First, the group structure that optimizes modularity within a given subgraph can depend on the number of edges in the entire graph in which the subgraph is embedded. Specifically, modularity is characterized by an intrinsic scale under which $Q$ is maximized when pairs of distinct groups having fewer than $\sqrt{2 l}$ edges (where $l$ is the total number of edges in the graph) are combined into single groups [4]. This phenomenon is apparent in ring graphs, i.e., connected graphs that consist of identical subgraphs each connected to exactly two other subgraphs by a single link. For example, in the graph shown in Figure 1 consisting of a ring of 15 squares, modularity is greater when adjacent squares are grouped together than when each square is a separate group.

A second weakness of modularity is that even when the resolution limit is not exceeded, modularity exhibits a bias towards groups of similar size. Intuitively, 
the sum of the square terms, $\left(l_{i} / l\right)^{2}$, representing the expected number of intragroup edges within community $i$ under the null model, is minimized, and $Q$ therefore maximized, when all $l_{i}$ are as nearly equal in size as possible.

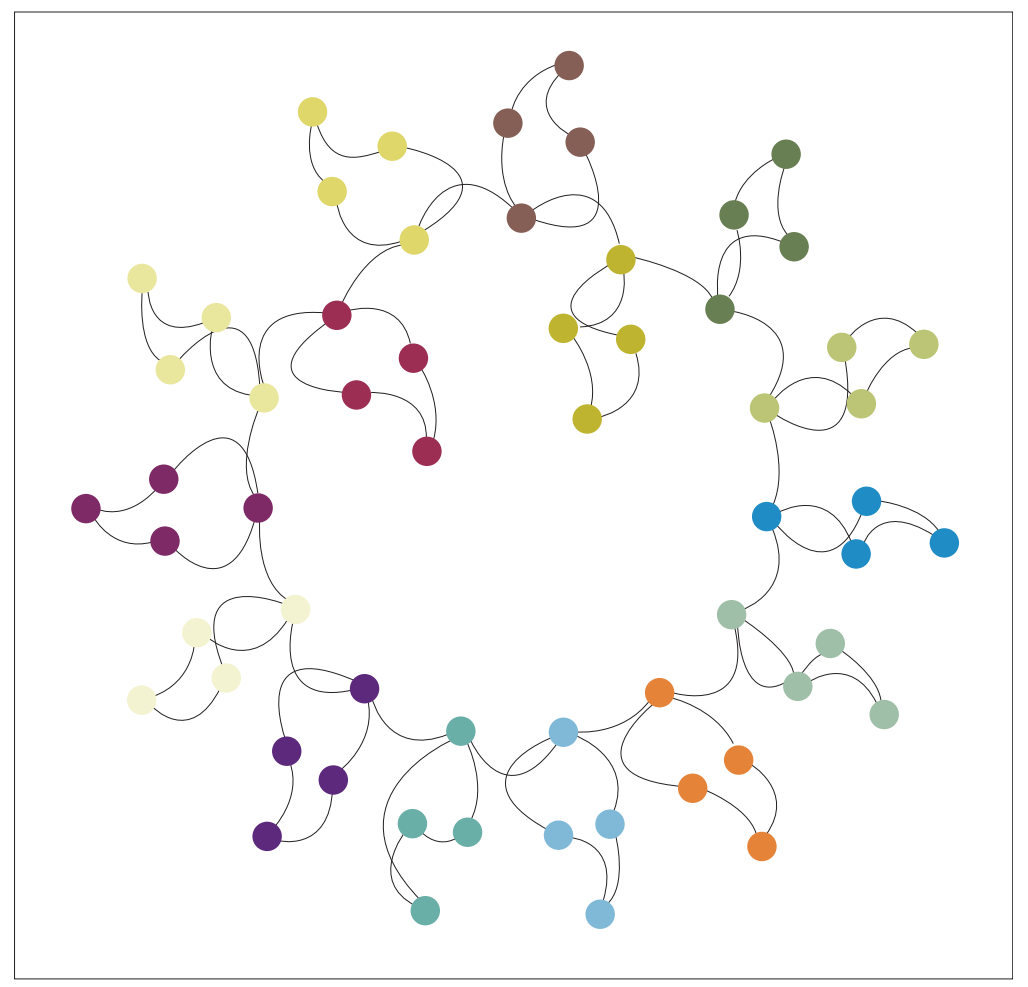

Fig. 1. Ring graph $R_{15,4}$ consisting of 15 communities, each containing 4 vertices.

One approach to the resolution limit of modularity is to apply modularity recursively, so that the coarse structure found at one level is refined at lower levels $[5] .^{2}$ An alternative approach is to substitute a different community-quality criterion for modularity.

One such alternative criterion for community quality that has recently been proposed, based on information theory, is minimizing description length [7-9]. In this approach, the quality of a given partition of a graph is a function of the complexity of the community structure together with the mutual information between the community structure and the graph as a whole. The best community structure is one that minimizes the sum of (1) the number of bits needed to represent the community structure plus (2) the number of bits needed to repre-

\footnotetext{
${ }^{2}$ See [6] for recent approach that addresses resolution limits by using an absolute evaluation of community structure rather than comparison to a null model.
} 
sent the entire graph given the community structure. Under this approach, the task of community detection consists of finding the community structure that leads to the minimum description length (MDL) representation of the graph, where description length is measured in number of bits.

The structure of the paper is as follows: Section 2 of this paper compares the compression approach used in two previous approaches to information-theoretic community detection and identifies a feature common to both that can lead to a bias toward combining distinct communities in large sparse graphs. An alternative encoding, termed SGE (Sparse Graph Encoding) that addresses this bias is proposed in Section 3. Section 4 describes the design of an empirical evaluation comparing the previous information-theoretic utility functions, SGE, and modularity on three classes of artificial data. The results of this experiment are set forth in Section 5.

\section{Minimum Description Length Encodings}

The intuition behind the minimum description length (MDL) criterion for community structure is that a partition of a graph that permits a more concise description of the graph is more faithful to the actual community structure than a partition leading to a less concise description. The best partition is the one that lends itself to the most concise description, that is, the encoding of the partition and of the graph given with the partition in the fewest bits. However, the minimum description length (MDL) criterion does not in itself specify how to encode either the community structure or the graph given the community structure. Indeed, the close connection between MDL and Kolmogorov complexity [10], which is undecidable, suggests that MDL may itself be undecidable.

The encoding algorithms of Rosvall and Bergstrom [7] (hereinafter "RB") and Chakrabarti [8] (hereinafter "AP," standing for "AutoPart") use quite different approaches to measuring the description length of community structures and graphs. However, RB and AP have in common that both are characterized by a resolution limit similar to that observed in modularity.

$\mathrm{RB}$ and AP decompose the task of encoding a graph and its community structure into similar steps, but they calculate the bits in each term differently. For the purposes of this comparison, the following notation will be followed:

$-n$ - the number of vertices in the graph

- $m$ - the number of groups

$-a_{i}$ - the number of vertices in group $i$

$-l$ - the total number of edges in the entire graph

- $l_{i}$ - the number of edges incident to group $i$

- $D_{i j}$ - a binary adjacency matrix between groups $i$ and $j$

- $n\left(D_{i j}\right)$ - the number of elements in adjacency matrix $D$

- $w\left(D_{i j}\right)$ - the number of 1 's in $D_{i j}$, i.e., the number of edges between groups $i$ and $j$

- $P\left(D_{i j}\right)$ - the density of 1's in $D_{i j}$, i.e., $\frac{w\left(D_{i j}\right)}{n\left(D_{i j}\right)}$ 
- $P^{\prime}\left(D_{i j}\right)$ - for a square matrix $D_{i j}$, the density of 1's ignoring the diagonal

- $H\left(D_{i j}\right)=-P\left(D_{i j}\right) \log \left(P\left(D_{i j}\right)\right)-\left(1-P\left(D_{i j}\right)\right) \log \left(1-P\left(D_{i j}\right)\right)$, i.e., the mean entropy of $D_{i j}$

- $H^{\prime}\left(D_{i j}\right)=-P^{\prime}\left(D_{i j}\right) \log \left(P^{\prime}\left(D_{i j}\right)\right)-\left(1-P^{\prime}\left(D_{i j}\right)\right) \log \left(1-P^{\prime}\left(D_{i j}\right)\right)$, i.e., the mean entropy of $D_{i j}$ if values on the diagonal of $D_{i j}$ are ignored

- B - a matrix representing for each pair of groups whether the pair is connected, i.e., $B_{i j}=1 \Longleftrightarrow w\left(D_{i j}\right)>0$

The encoding schemes used in RB and AP are as follows:

1. Bits needed to represent the number of vertices in the graph. Since this term doesn't vary with differing community structure, it is irrelevant to the choice between different community structures and can be ignored.

2. Bits needed to represent the number of groups.

- RB. Not explicitly represented.

- AP. $\log ^{*}(m) \cdot \log ^{*}(x)=\log _{2}(x)+\log _{2} \log _{2}(x)+\ldots$ where only positive terms are included in the sum. This series is apparently intended to represent the mean coding length of integers given that the probability of an integer of a given length is a monotonically decreasing function of the integer's length, i.e., longer integers are less probable, but no maximum length is known [17].

3. Bits needed to represent the association between vertices and groups

- RB. $n \log (m)$. The rationale appears to be that for each of the $n$ vertices, $\log (m)$ bits are needed to identify the group to which the vertex belongs.

- AP. If the groups are placed in decreasing order of length, i.e., $a_{1} \geq a_{2} \geq$ $\ldots \geq a_{m} \geq 1$,

$$
\sum_{i=1}^{m-1}\left\lceil\log \left(\bar{a}_{i}\right)\right\rceil
$$

where $\bar{a}_{i}=\left(\sum_{t=1}^{m} a_{t}\right)-m+i$.

4. Bits needed for the group adjacency matrix, i.e., the number of edges between pairs of groups.

- RB. $\frac{1}{2} m(m+1) \log (l)$. The first term $\left(\frac{1}{2} m(m+1)\right)$ represents the number of pairs of groups, and the second term $(\log (l))$ the number of bits needed to specify the number of edges between any pair of groups.

$-\mathrm{AP}$.

$$
\sum_{1<i, j<m}\left\lceil\log \left(a_{i} a_{j}+1\right)\right\rceil
$$

This expression sums for every pair of groups sufficient bits to represent the number of edges between that pair.

5. Bits needed to represent the full adjacency matrix for vertices, given the group structure represented in terms 2-4.

- RB.

$$
\log \left(\prod_{i=1}^{m}\left(\begin{array}{c}
a_{i}\left(a_{i}-1\right) / 2 \\
w\left(D_{i i}\right)
\end{array}\right) \prod_{i<j}\left(\begin{array}{c}
a_{i} a_{j} \\
w\left(D_{i j}\right)
\end{array}\right)\right)
$$


The expression following the first product sign represents the number of ways to choose the actual pairs that are connected within a single group from the set of all possible pairs. The expression following the second product sign is the number of ways to choose the actual pairs between vertices in two different groups from the set of possible edges between vertices in those groups.

- AP.

$$
\sum_{i=1}^{m} \sum_{j=1}^{m} a_{i} a_{j} H\left(D_{i j}\right)
$$

For each pair of groups, the entropy of the adjacency matrix for that pair, i.e., the size of the matrix times its the mean entropy.

$\mathrm{RB}$ and AP clearly calculate each term quite differently. In general, RB uses encodings that are much larger than those used in AP. However, a key similarity is in term 4 , the bits needed to encode the number of edges between pairs of groups. In both RB and AP at least one bit is required for each pair of groups regardless of how few groups are actually connected (i.e., how few pairs of groups have at least one edge from a vertex in one to a vertex in the other). The number of bits arising from this term therefore increases with the square of the number of groups, regardless of the sparsity of their interconnections. One would expect that for sufficiently large graphs with sparse community structure the savings in term 4 from combining groups would be greater than the added cost in term 5 of specifying the vertex adjacencies for the resulting relatively sparse group, and that this would lead to conflation of distinct groups similar to that observed when modularity is used as a community quality function. As discussed in the evaluation below, this conflation is in fact observed. For example, the number of bits required to encode the graph shown in Figure 1 is lower under both the $\mathrm{RB}$ and AP procedures if some pair of adjacent groups are combined, yielding 14 communities, than if it is divided into 15 equal communities.

\section{$3 \quad$ Sparse Graph Encoding (SGE)}

The observations that RB and AP (1) assign at least one bit per pair of communities, regardless of how few are actually connected and (2) conflate distinct groups in large sparse graphs (as shown experimentally below) suggests the hypothesis that an encoding in which the bits required to encode the number of edges between pairs of groups grow more slowly than the square of the number of groups would be less prone to the resolution limit. Sparse Graph Encoding (SGE) is an encoding scheme designed to test this hypothesis.

The key idea is to encode the group adjacency matrix using two terms. The first term encodes, for each pair of groups, whether the groups are connected. The number of bits required for this is equal to the entropy of B, the binary matrix representing for each pair of groups whether those groups are connected. The mean entropy of B is at most 1.0, if each group is randomly connected to exactly half the others. If few, or most, groups are connected to one another, the 
mean entropy is less than 1.0, and the total entropy is therefore less than the square of the number of groups.

Moreover, the number of bits needed to represent B can be further reduced by noting that the value of B's diagonal need not be explicitly represented because it can be determined from the number of nodes in each group. Singleton groups have no within-group edges (assuming that self-loops are prohibited) and groups with more than one element must have at least one within-group edge (if there are no within-group edges, the density of within-group edges cannot be higher than the density of between-group edges, the basic characteristic of a group).

The bits needed to represent $\mathrm{B}$ are therefore:

$$
m(m-1) H^{\prime}(B)
$$

where $H^{\prime}(B)=-P^{\prime}(B) \log \left(P^{\prime}(B)\right)-\left(1-P^{\prime}(B)\right) \log \left(1-P^{\prime}(B)\right)$ and $P^{\prime}(B)$ is the density of $1^{\prime}$ 's in $\mathrm{B}$, ignoring the diagonal.

The second term contains, for each connected pair, the number of bits needed to represent the number of edges between that pair (the second sum is needed if, as we assume, edges from a vertex to itself are forbidden):

$$
\sum_{i \neq j \wedge w\left(D_{i j}\right) \geq 0} \log \left(a_{i} a_{j}\right)+\sum_{i=j \wedge w\left(D_{i j}\right)>0} \log \left(a_{i}\left(a_{j}-1\right)\right)
$$

If the cost of representing the group adjacent matrix is calculated as expression $2+$ expression 3 , the cost will grow with the number of connected pairs rather than with the total number of pairs.

SGE employs several additional minor modifications to further reduce the description length. The entire calculation is as follows:

1. Bits needed to represent the number of vertices in the graph. As with RB and AP, these bits are ignored.

2. Bits needed to represent the number of groups. The $\log ^{*}$ function of $[17]$ used in AP is predicated on the assumption that no maximum integer size is known a priori. Here, however, the maximum number of groups is bounded by both the machine word size and the virtual memory size of the machine on which the algorithm is executed. Therefore, SGE uses instead RB's calculation:

$$
\log (m)
$$

3. Bits needed to represent the association between vertices and groups. No group can contain more than $n-m+l$ vertices (since each group must have at least one vertex). Accordingly, the following expression contains sufficient bits to represent the number of vertices in all $\mathrm{m}$ groups:

$$
m \log (n-m+1)
$$

4. Bits needed for the group adjacency matrix, i.e., the number of edges between pairs of groups. As discussed above, the number of bits is:

$$
H^{\prime}(B)+\sum_{i \neq j \wedge w\left(D_{i j}\right)>0} \log \left(a_{i} a_{j}\right)+\sum_{i=j \wedge w\left(D_{i j}\right)>0} \log \left(a_{i}\left(a_{j}-1\right)\right)
$$


5. Bits needed to represent the full adjacency matrix for vertices given the group structure represented in terms 2-4. This consists, for every pair of groups $i$ and $j$, of size of the $i, j$ adjacency matrix, $a_{i} a_{j}$, times the entropy per entry in the corresponding binary matrix, $H\left(D_{i j}\right)$. This is equivalent to the AP calculation, shown above:

$$
\sum_{i=1}^{m} \sum_{j=1}^{m} a_{i} a_{j} H\left(D_{i j}\right)
$$

In summary, the relationship between SGE, RB, and AP is as follows:

1. Bits needed to represent the number of vertices in the graph. Ignored as in $\mathrm{RB}$ and AP.

2. Bits needed to represent the number of groups. Follows RB.

3. Bits needed to represent the association between vertices and groups. Uses an expression with fewer bits than that used in $\mathrm{RB}$, and that is simpler than that used in AP.

4. Bits needed for the group adjacency matrix. The primary novelty of SGE, in that for sparse adjacency matrices this term grows more slowly than the square of the number of groups.

5. Bits needed to represent the full adjacency matrix for vertices. Follows AP.

\section{Empirical Evaluation}

The previous section suggested that a graph encoding in which the calculation of the number bits required to represent a group adjacency matrix was reduced from an expression that grows as the square of the number of groups, as in RB and AP, to an expression that grows in proportion to the number of pairs of connected groups, as in SGE, would reduce or eliminate any resolution limit in sparsely connected graphs. This hypothesis was tested by comparing the communities found by optimizing RB, AP, SGE, and modularity on three different artificial data sets.

To avoid conflating the effect of a utility function with the behavior of a search strategy, it was necessary to compare alternative utility functions using a single common search strategy. Accordingly, a single search function was applied to all for utility functions in the experimental evaluation: the greedy divisive hierarchical clustering algorithm of Newman \& Girvan (2004) [11]. In the Newman \& Girvan procedure, the edge with the highest betweenness centrality is iteratively removed, and the partition in the resulting sequence having the optimal value under the utility function was returned as the community structure. Using a single search strategy removes the potentially confounding disparity of the search algorithms used in published descriptions of RB, AP, and modularity.

\subsection{Evaluation Criteria}

Various objective functions have been proposed for evaluating the quality of a proposed community structure given the actual correct community structure, 
including the Rand index [23], the adjusted Rand index [24], and f-measure. There is no consensus regarding the most informative objective function. In this evaluation, f-measure was selected since its use in information retrieval has made it familiar to a wide range of researchers.

The intuition underlying the use of f-measure is that group structure can be expressed as a relation $c(G)=\left\{\left\langle v_{i}, v_{j}\right\rangle \mid \exists g \in G \wedge v_{i}, v_{j} \in g\right\}$, that is, the community structure can be represented by specifying for each pair of vertices whether that pair is in the same group. The similarity between the proposed group structure and the actual group structure can be evaluated by comparing $c$ (proposed) with $c$ (actual). One way to make the comparison is to view each pair in c(proposed) that is also in c(actual) as a true positive, whereas each pair in c(proposed) that is not in c(actual) is a false positive. Under this view, recall and precision can be defined as follows:

$$
\begin{aligned}
& - \text { Recall }=\frac{\mid c(\text { proposed })|\cap| c(\text { actual }) \mid}{\mid c(\text { actual }) \mid} \\
& - \text { Precision }=\frac{\mid c(\text { proposed })|\cap| c(\text { actual }) \mid}{\mid c(\text { proposed }) \mid}
\end{aligned}
$$

F-measure is the harmonic mean of recall and precision:

$$
\text { - F-measure }=\frac{2 * \text { recall } * \text { precision }}{\text { recall }+ \text { precision }}
$$

\subsection{Experimental Procedure}

Three experiments were performed, each with a different type of artificial graph. The first, ring graphs, are characterized by the sparsity of connections between groups observed in many large-scale real-world graphs [20]. The second, uniform random graphs, has been used in a number of evaluations of community-detection algorithms. The third, embedded Barabasi-Albert Graphs, consists of communities generated by preferential attachment [20] embedded in a random graph. Fifty trials were performed under each experimental condition for uniform random and EBA graphs. There is no randomness in the construction of ring graphs, so a single trial was sufficient.

Experiment 1: Ring graphs. Ring graph $R_{m, c}$ comprises $m$ communities, each consisting of a ring of $c$ vertices, connected to two other communities, each by a single link, such that all communities are connected. Ring graphs are similar to the clique rings of [4] but differ in that the individual communities are themselves rings rather than cliques. For example, Figure 1 depicts ring graph $R_{15,4}$.

The evaluation compared RB, AP, SGE, and modularity on 91 ring graphs for which $\langle m, c\rangle \in\{4 \ldots 16\} \times\{3 \ldots 9\} .^{3}$ Strikingly different behavior was observed

\footnotetext{
${ }^{3}$ Note that for $m, c>3$ ring graphs contain no triangles. Therefore, community detection techniques based on clustering coefficient, e.g., [18], are ineffective for finding communities in such ring graphs.
} 


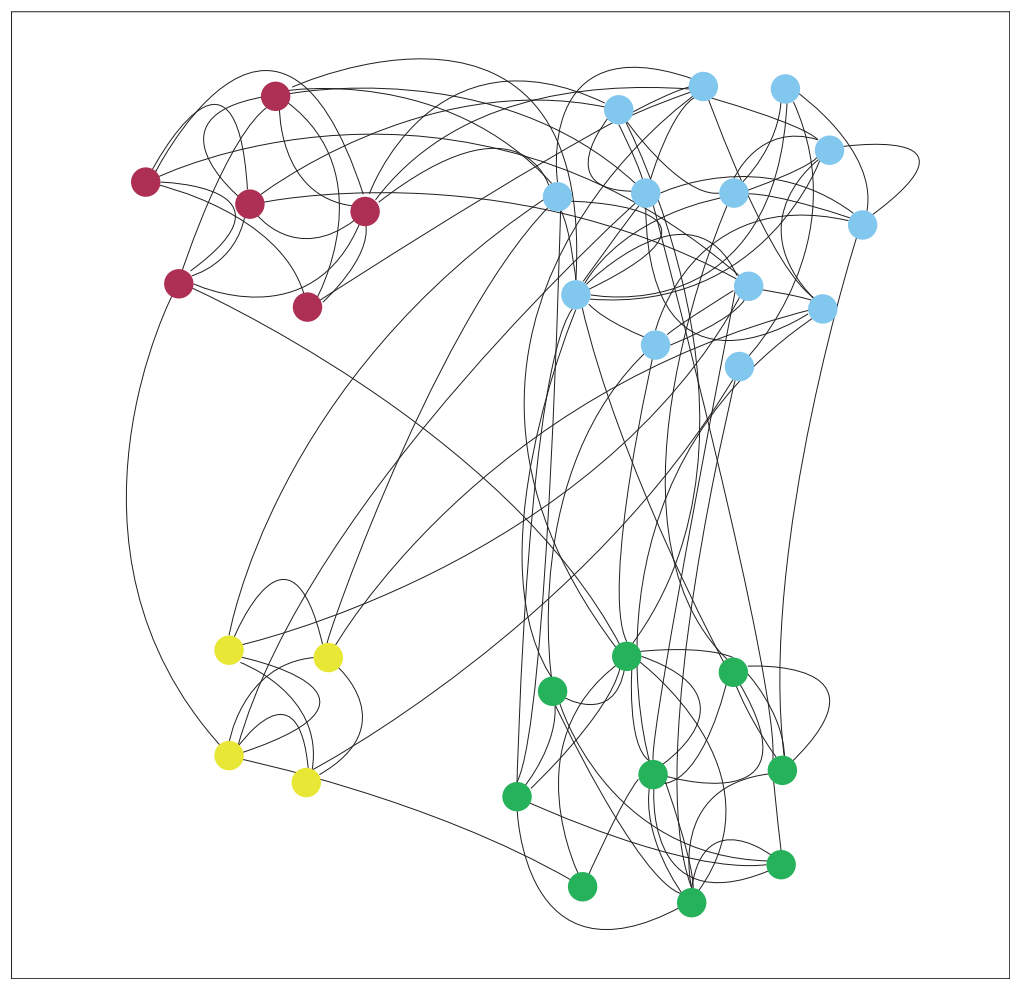

Fig. 2. A uniform random graph with 32 vertices, 4 groups, size ratio 1.25 , and io ratio 0.67 . 


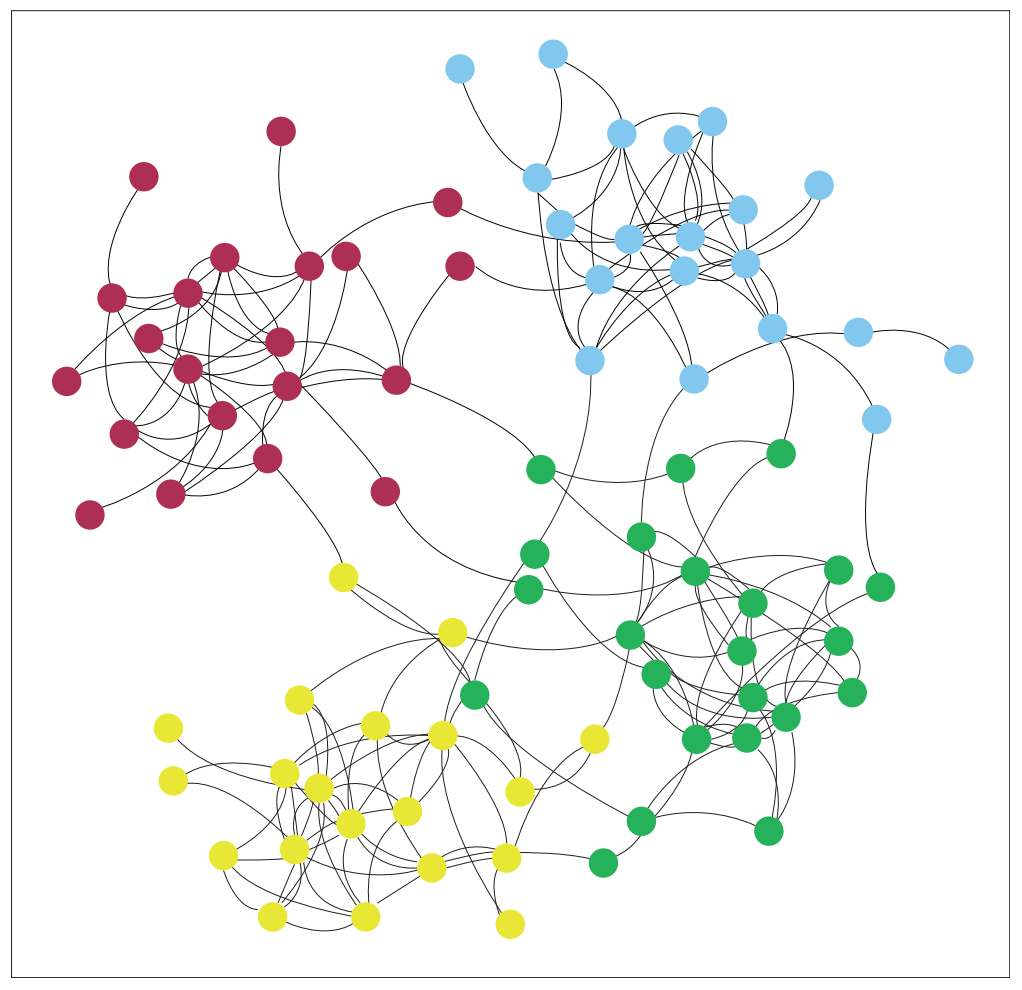

Fig. 3. An Embedded Barabasi-Albert (EBA) graph with 4 communities, each with 5 initial vertices per community, 3 new edges per time step, 10 time steps, and 25 singleton-group edges. 
among the four community-structure utility functions. Optimizing SGE led to the correct partitions in all but two ring graphs, but RB and AP found no correct partitions. Optimizing modularity led to correct partitions only for those graphs below the resolution threshold identified by [4].

- SGE. The partition having the optimal (lowest) SGE had the correct partition (i.e., no separate communities were conflated) in every graph except for $R_{4,3}$ and $R_{13,3}$ In other words, the correct community structure was found in 89 out of 91 ring graphs.

- RB and AP. No community structure was found by optimizing either RB or AP. The partition having the optimal (lowest) value for RB and AP contained at least one pair of communities that were grouped together in every ring graph tested.

- Modularity. Optimizing modularity led to incorrect community structure for rings of more than 8 triangles, more than 10 squares, more than 11 pentagons, or more that 13 hexagons or heptagons. In other words, the correct partitions were obtained with modularity only for rings and communities of the following sizes:

- $R_{4,3}-R_{8,3}$

- $R_{4,4}-R_{10,4}$

- $R_{4,5}-R_{11,5}$

- $R_{4,6}-R_{13,6}$

- $R_{4,7}-R_{13,7}$

- $R_{4,8}-R_{16,8}$

- $R_{4,9}-R_{16,9}$

This evaluation confirmed empirically the existence of the resolution limit for modularity derived formally in [4]. The evaluation also showed the surprising result that optimizing $\mathrm{RB}$ and $\mathrm{AP}$ leads to even more conflation of distinct communities than does modularity. The observation that optimizing SGE led to the correct community structure provides confirmation for the hypothesis that the conflation of communities in RB and AP arises from term 4, which uses more bits than necessary to represent the number of edges connecting groups in sparse graphs. Substituting rings of cliques for rings of graphs that are themselves rings leads to almost identical results to those described here.

Experiment 2: Uniform random graphs. A common data set for testing community-extraction algorithms consists of random networks of 128 vertices divided into 4 communities with average vertex degree of $16[11,16,19]$. In this experiment, the relative size of the communities was controlled by a size ratio parameter $s$ such that if the communities were placed in ascending order, $\frac{\left|a_{i+1}\right|}{\left|a_{i}\right|}=$ $s$, where $a_{i}$ is the $i t h$ communities. The connections among the vertices were determined by the average vertex degree $d$ and in/out ratio $i$ such that the average number of within-community edges incident to each vertex was $i * d$ and the average number of cross-community edges incident to each vertex was $(1-i) * d$. For example, Figure 2 shows a uniform random graph with $s=1.25$ 


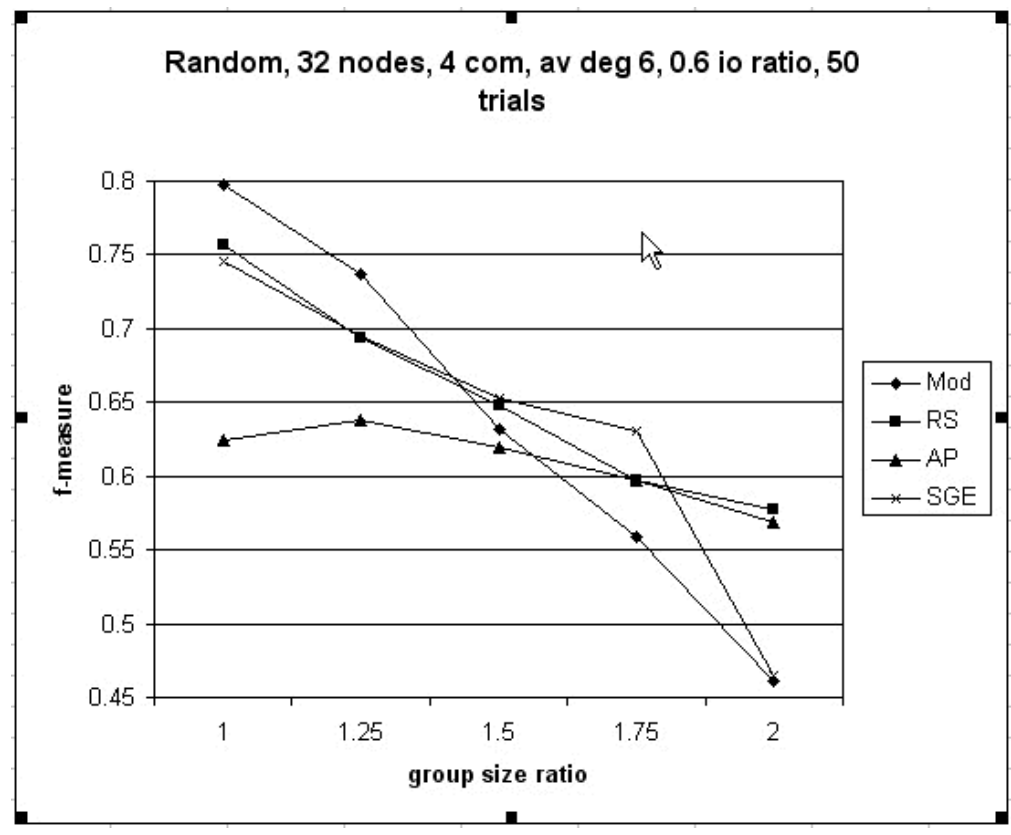

Fig. 4. F-measure for uniform random graphs with $\mathrm{i}=0.6$ (weak community structure).

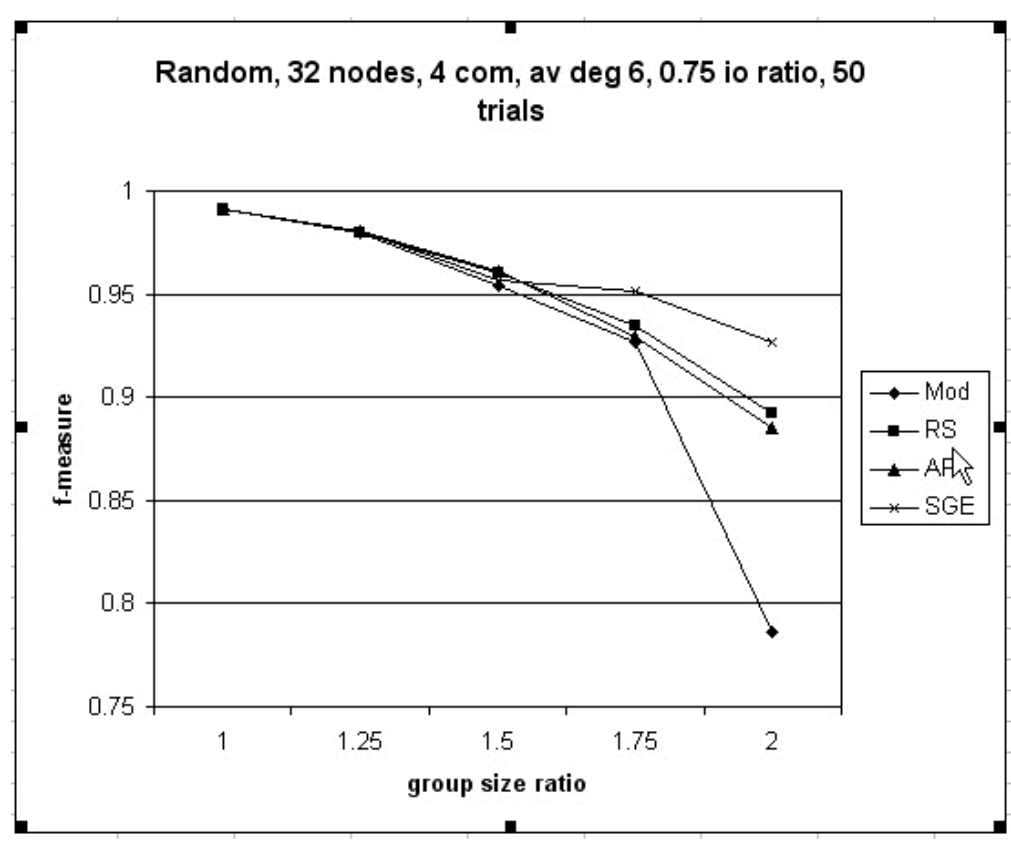

Fig. 5. F-measure for uniform random graphs with $\mathrm{i}=0.75$ (moderate community structure). 


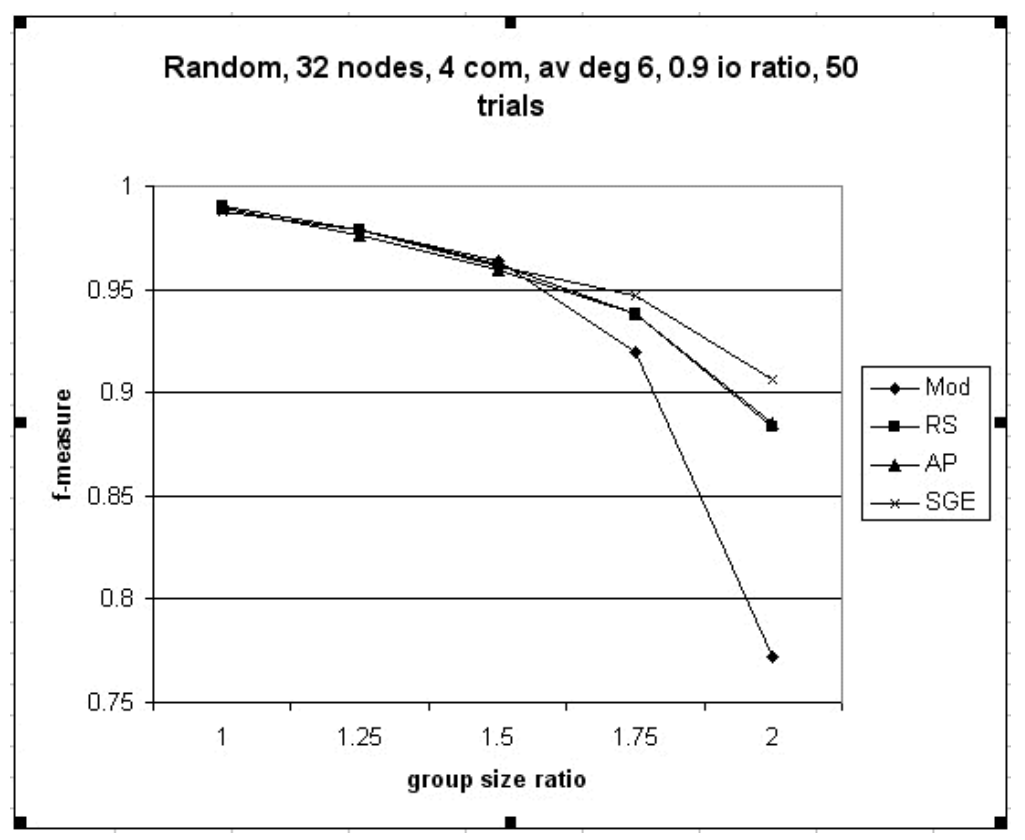

Fig. 6. F-measure for uniform random graphs with $\mathrm{i}=0.9$ (strong community structure).

and $i=0.6$. Tests were performed for each combination of $n=32, m=4, d=$ $6, s \in\{1.0,1.25,1.5,1.75,2.0\}$, and $i \in\{0.6,0.75,0.9\}$.

Figures 4,5 , and 6 show the results of the 4 algorithms on uniform graphs for $i \in\{0.6,0.75,0.9\}$ respectively. For $i \in\{0.75,0.9\}$, in which the community structure is relatively distinct, all four algorithms led to similar results except when the size ratio $s$ was equal to 2.0 (i.e., the sizes of the groups were highly skewed). Under these circumstances, modularity led to much lower f-measure than the other algorithms. When $i$ was equal to 0.6 (i.e., the community structure was relatively unclear) modularity was best and AP worst for low size ratio, and $\mathrm{RB}$ and AP were best for high size ratio. These results are consistent with [7], which showed better performance for RB than modularity for skewed community sizes, but comparable performance when community sizes were equal.

Experiment 3: Embedded Barabasi-Albert Graphs. A wide range of naturally occurring graphs, including those mentioned in the introduction (the Internet, biochemical pathways, social networks) exhibit a power-law degree distribution that is not present in uniform random graphs [20-22]. However, few such "scale-free" graphs are annotated with correct community structure. The third data set consisted of communities with scale-free structure embedded in a sparse random graph. Each graph consists of $m$ communities generated by the Jung 1.74 implementation of the Barabasi-Albert preferential attachment algorithm, each starting with $i$ initial vertices in each community, with $e$ new 


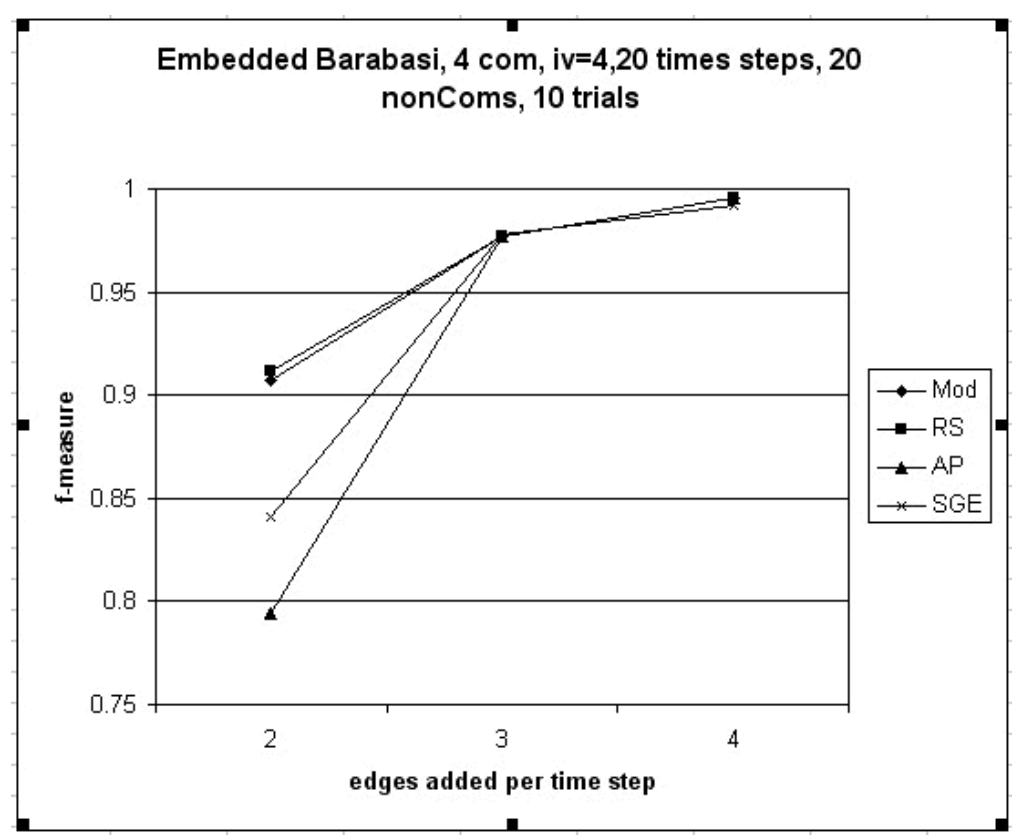

Fig. 7. F-measure for embedded Barabasi-Albert graph with 2-4 edges added per time step.

edges per time step following the preferential attachment rule of [20] for each of $t$ time steps, together with $c$ singleton-group vertices. The singleton-group vertices were connected to 1 ...e vertices randomly selected from the entire graph, i.e., including both community and singleton-group vertices. The graphs used tor testing had 4 communities, 4 initial vertices per community, $2-4$ edges added per time step, 20 time steps, and 25 singleton-group vertices. For example, Figure 3 depicts an EBA graph with 3 edges added per time step. In evaluating EBA graphs, singleton-group vertices were ignored, regardless of whether they were grouped into new communities or added to existing communities.

As shown in Figure 7, the behavior of all four algorithms was quite similar when the number of edges added per time step was 3 or 4 , which leads to relatively densely connected graphs. When only 2 edges were added per time step (i.e., the communities where quite sparse), AP's performance was much worse, and SGE's somewhat worse, than that of the other two algorithms.

\section{Conclusion}

The empirical evaluation demonstrated that $\mathrm{RB}$ and $\mathrm{AP}$ conflate distinct communities in ring graphs, and that changing the calculation of the number of bits needed to represent the group adjacency matrix eliminated this conflation over the range of ring graphs tested. Ring graphs are artifacts not likely to occur 
in many real-world graphs of interest, but many real-world graphs are like ring graphs in having very sparse group adjacency matrices (i.e., communities with links to few other communities). The ring-graph experiment suggests that RB and AP may perform even more poorly than modularity in such graphs.

SGE's description length calculation did not entirely eliminate resolution limits in clustering. For example, SGE combines adjacent communities in extremely large rings, such as $R_{100,4}$. Moreover, SGE combines adjacent communities in $R_{3,4}$ and $R_{13,3}$. Thus, it appears that SGE's bit encoding is not optimal even in sparse graphs.

No one algorithm consistently outperformed the others in EBA or uniform random graphs, but modularity was consistently worse than the MDL algorithms on highly skewed uniform random graphs, and AP and SGE had lower performance than the others on sparse EBA graphs. Neither uniform random graphs nor EBA graphs have the sparse group adjacency matrices that characterize ring graphs, so most errors consist of assigning a vertex to the wrong community rather than combining two communities that should remain distinct. Under these circumstances, SGE's representation of the group adjacency matrix confers no particular benefit.

While MDL is clearly a powerful tool for identifying community structure, there are many options for MDL encodings, and the consequences of each choice can be difficult to anticipate. SGE demonstrates that the resolution limits of RB and AP in graphs with sparse group adjacency matrices can be easily addressed, but the fact that SGE did not outperform RB or RB on other types of graphs suggests that considerable subtlety is required to identify the MDL encoding most effective over a wide range of graph and community types.

\section{Acknowledgments}

This work was funded under contract number CECOM W15P7T-08-C-F600. The MITRE Corporation is a nonprofit Federally Funded Research and Development Center chartered in the public interest.

\section{References}

1. Newman, M.E.J.: Fast algorithm for detecting community structure in networks. Physical Review E 69 (2004) 066133

2. Ganti, V., Ramakrishnan, R., Gehrke, J., Powell, A.L., French, J.C.: Clustering large datasets in arbitrary metric spaces. In: Proceedings of the 15th IEEE International Conference on Data Engineering, Sydney (1999) 502-511

3. Koutsourelakis, P., Eliassi-Rad, T.: Finding mixed-memberships in social networks. In: Papers from the 2008 AAAI Spring Symposium on Social Information Processing, Technical Report WW-08-06, AAAI Press (2008) 48-53

4. Fortunato, S., Barthelemy, M.: Resolution limit in community detection. PROC.NATL.ACAD.SCI.USA 104 (2007) 36

5. Ruan, J., Zhang, W.: Identifying network communities with a high resolution. PhysRevE (2007) 
6. Ronhovde, P., Nussinov, Z.: An improved potts model applied to community detection. physics.soc-ph (2008)

7. Rosvall, M., Bergstrom, C.: An information-theoretic framework for resolving community structure in complex networks. Proc Natl Acad Sci USA 104(18) (May 2007) 7327-7331

8. Chakrabarti, D.: Autopart: Parameter-free graph partitioning and outlier detection. In: Proceedings of the European Conference on Machine Learning and Practice of Knowledge Discovery in Databases. (2004) 112-124

9. Sun, J., Faloutsos, C., Papadimitriou, S., Yu, P.: Graphscope: parameter-free mining of large time-evolving graphs. In: KDD '07: Proceedings of the 13th ACM SIGKDD international conference on Knowledge discovery and data mining, ACM (2007) 687-696

10. Wallace, C.S., Dowe, D.L.: Minimum message length and Kolmogorov complexity. The Computer Journal 42(4) (1999) 270-283

11. Newman, M.E., Girvan, M.: Finding and evaluating community structure in networks. Physical review. E, Statistical, nonlinear, and soft matter physics 69(2 Pt 2) (February 2004)

12. Tasgin, M., Bingol, H.: Community detection in complex networks using genetic algorithm. In: ECCS '06: Proc. of the European Conference on Complex Systems. (2006)

13. Rattigan, M.J., Maier, M., Jensen, D.: Graph clustering with network structure indices. In: ICML '07: Proceedings of the 24th international conference on Machine learning, New York, NY, USA, ACM (2007) 783-790

14. Donetti, L., Muoz, M.: Detecting net-work communities: a new systematic and efficient algorithm. Journal of Statistical Mechanics: Theory and Experiment 10012 (2004) 1-15

15. Zhang, H., Giles, C.L., Foley, H.C., Yen, J.: Probabilistic community discovery using hierarchical latent gaussian mixture model. In: AAAI'07: Proceedings of the 22nd national conference on Artificial intelligence, AAAI Press (2007) 663-668

16. M., R., C., B.: An information-theoretic framework for resolving community structure in complex networks. PNAS 104(7327) (2007)

17. Rissanen, R.: A universal prior for integers and estimation by minimum description length. The Annals of Statistics 2 (1983) 416-431

18. Du, N., Wu, B., Pei, X., Wang, B., Xu, L.: Community detection in large-scale social networks. In: WebKDD/SNA-KDD '07: Proceedings of the 9th WebKDD and 1st SNA-KDD 2007 workshop on Web mining and social network analysis, New York, NY, USA, ACM (2007) 16-25

19. Bagrow, J.: Evaluating local community methods in networks. J. Stat. Mech. 2008(05) (May 2008) P05001

20. Barabasi, A., Albert, R.: Emergence of scaling in random networks. Science $\mathbf{2 8 6}$ (Oct 15 1999) 509-512

21. Clauset, A., Shalizi, C.R., Newman, M.E.J.: Power-law distributions in empirical data (2007) cite arxiv:0706.1062 http://www.santafe.edu/ aaronc/powerlaws/.

22. Clauset, A., Shalizi, C., Newman, M.: Power-law distributions in empirical data. SIAM Review 51(4) (2009) 661-703

23. Rand, W.M.: Objective Criteria for the Evaluation of Clustering Methods. Journal of the American Statistical Association 66(336) (1971) 846-850

24. Hubert, L., Arabie, P.: Comparing partitions. Journal of Classification 2 (1985) $193-218$ 